\title{
Saving of Etalons in Image Processing Systems Based on the Parallel Shift Technology
}

\author{
Stepan Bilan ${ }^{1}$, Sergey Yuzhakov ${ }^{2}$ and Sergii Bilan ${ }^{3}$ \\ ${ }^{1}$ State Economy and Technology University of Transport, Kiev, Ukraine \\ ${ }^{2}$ Haysin merged state tax inspection of Main governance of SFS in Vinnitsa region, Haysin, Ukraine \\ ${ }^{3}$ Win-Interactive LLC, Vinnytsia, Ukraine \\ 1'bstepan@ukr.net, ${ }^{2}$ yserg74@meta.ua, ${ }^{3}$ belan@svitonline.com
}

\begin{abstract}
This article describes variants saving of etalons in image processing systems based on the parallel shift technology. The parallel shift technology can be used in hybrid image processing systems.
\end{abstract}

Keywords: parallel shift technology, function of the area of intersection, Fourier transformation.

\section{Introduction}

The use of hybrid systems to model any process is more productive than the use of systems that are based on a one method of information processing [1]. This article explains process description and saving of etalons in the systems which based on the use of multiple image transformations.

The digital image processing most widely used to manipulate with video information. This process is characterized by a description of the visual scene as a set of pixels. In this case, the formation of this set at digitization images are rather simple process. The main burden in image processing is to analyze a set of pixels [2]. The number of computational operations increases with discreteness digitized image.

It is proposed to describe visual scene also as a set of features for building a hybrid systems of image processing $[3,4]$. The number of such functions must be significantly smaller than the number of digitized image pixels. That is, the set of pixels have to unite on certain features. The area of the specific image parts can be the unifying parameter. Its dynamic changes should be carried out to transform static area parameter to the function. The performance of certain image processing tasks is enhanced by reducing the computational operations that occurs as a result of reducing the number of objects for analysis.

Means of obtaining the function of the area of intersection (FAI) is a parallel shift technology (PST). PST is a process of interaction object that describes by $n$-dimensional characteristic features vector (CF), with a copy of which changes only one CF in comparison to the original. As a result, certain way got a superposition of the pair, which is different from the original object and can be used for further analysis. For example, the sum of the sinusoidal signal-copy which shifted in the phase space and its initial signal is different from a sinusoid. When phase shift is $\pi$, the sum will be zero. The change one of coordinates is using to obtain the function of the area of intersection. The result of the superposition of the original image and its parallel shifted copy is the FAl of these objects. A set of values of intersection area for each change of the coordinates is the FAI.

DOI : $10.14738 /$ aivp.26.772 
FAl is a function depending on intersection area of the original image and its copies which parallel shifted in the chosen direction, of the shift distance. The process of determining the area and parallel shift easily implemented using simple circuit solutions (Belan S. \& Belan N., 2012).

FAl $(x)$ is determined as the area of intersection of the image (Figure 1,a) and its copy, that is shifted in a parallel direction $\phi$ (Figure 1,b).

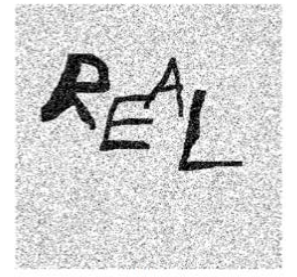

a) initial image

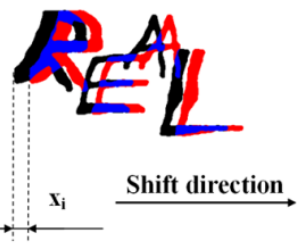

b) shift process

Figure 1: Image shift for FAl formation

Obtaining a pair of FAl by two orthogonal shifts (Figure 2).

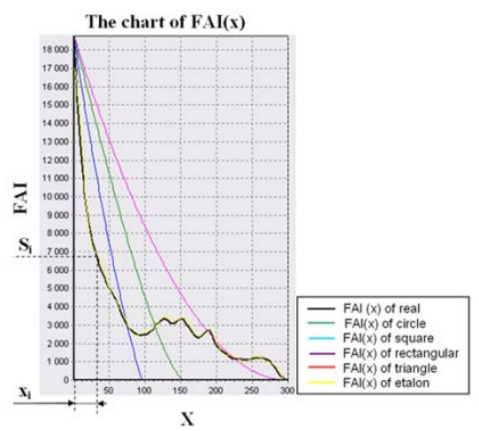

a) $\operatorname{FAI}(x)$

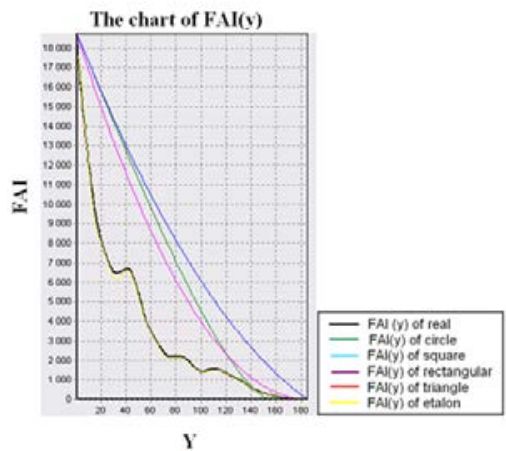

b) $\mathrm{FAI}(\mathrm{y})$

Figure 2: The functions of the area of intersection

In previous publications on this topic describes a variety of methods and algorithms for video processing using image transformation based parallel shift technology (Belan S. \& Yuzhakov S., 2013). This article deals with the variants saving of etalon data.

\section{Variants Saving of Etalon Data}

One of the characteristics of image processing with PST is preservation etalons in the form of a certain etalon surfaces created by the function of the area of intersection in the direction of $\phi$ to $\phi+$ $\pi$. Direction $\phi$ is the initial scan direction of image in the creation of etalon. It can be any direction. Only set of FAl reflects information about the shape of a particular object. Whatever kind of additional indicators were not used in the algorithms of image processing system to speed the processing steps, the final step is always the comparing real and etalon FAI.

The etalon surfaces may be stored as digitally and in analog form. Saving of etalon surfaces in digital form requires a large amount of hardware costs and requires additional processing facilities functions in comparison (requires scaling).

Variant of creating etalons for the reduction of the required information can be by saving of reduced etalon copies of images in the database. In this case, the values of etalon FAl can be generated from the reduced etalon directly in the detailed stage of recognition process. This method of saving will reduce the number of required hardware. But in this case it is increased the error in the recognition process. In addition, it does not eliminate the need for scaling functions. 
Stepan Bilan, Sergey Yuzhakov, Sergii Bilan; Saving of Etalons in Image Processing Systems Based on the Parallel Shift Technology. Advances in I mage and Video Processing, Volume 2 No 6, Dec (2014); pp: 36-41

Another method of etalons saving is to replace the etalons FAl by auxiliary functions which dependent on the input function of the area of intersection. At the stage of software simulation was used fast Fourier transformation (FFT). This version is simple in realization. It significantly reduce hardware costs and does not require additional processing methods of functions. Quantity of information required to store the etalon surface in the form of auxiliary function elements which depend on the number of etalons in the database.

Saving etalon surfaces in analog form can carry a highly accurate reflection of FAl. Using holography may be a variant of such saving. But the methods of processing such data description developed insufficiently. Image processing based on PST induces to development of new methods of information saving.

Procedure for the preparation of comparison etalon and real FAl in different variants of saving etalon surfaces is shown in Figure 3.

When using this method of describing images in analog computers is not compulsory digitization of FAl which obtained from the holography of etalon surfaces.

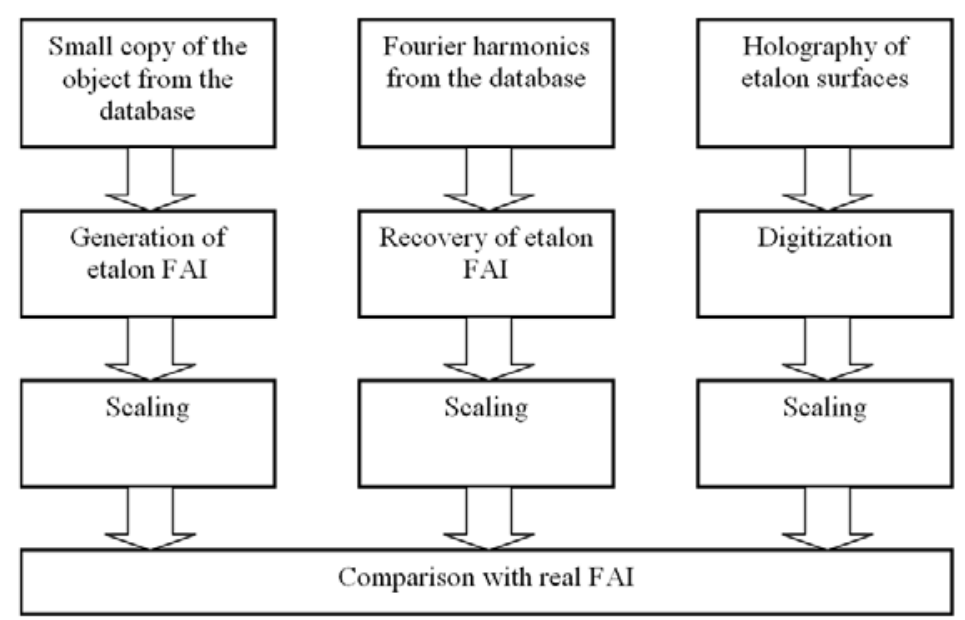

Figure 3: Procedure for the preparation of comparison etalon and real FAl in different variants of saving etalon surfaces

\section{Transformation FAI to Fourier Series}

A possible solution to the problem of etalon FAl saving is the use of Fourier transformation. As we know, such a transformation carries complete information about the function. The number of the Fourier series harmonics, which is necessary for saving the etalon surface, depending on the number of etalons. Normalization is required to unify the size values of harmonics. To achieve this, every element of series must be divided by the value of the input image area. In this case the value of all harmonics except the first are close to unity, which simplifies the storage of information in databases.

The following formulas for the normalized harmonics is used in constructing the Fourier series that reflect etalons FAI.

$$
\begin{gathered}
\text { FFTN }_{0}=\frac{1}{S_{0}} \frac{1}{\sqrt{X_{\text {max }}}} \sum_{j=0}^{X_{\text {max }}-1} F A I(j), \\
\text { FFTN }_{i}=\frac{1}{S_{0}} \frac{\sqrt{2}}{\sqrt{X_{\text {max }}}} \sum_{j=0}^{X_{\text {max }}-1} F A I(j) \cdot \cos \left(\frac{\pi \cdot i \cdot(2 \cdot j+1)}{2 \cdot X_{\text {max }}}\right)
\end{gathered}
$$


The value of the original image area $\left(\mathrm{S}_{0}\right)$ and maximal shift $\left(\mathrm{X}_{\max }\right)$ are basic FAI parameters. Reverse recovery FAl occurs by the formula 3 .

$$
F A I(j)=S_{0} \cdot\left(\frac{F F T N_{0}}{\sqrt{X_{\max }}}+\frac{\sqrt{2}}{\sqrt{X_{\max }}} \sum_{i=1}^{X_{\max }-1} \operatorname{FFTN}_{i} \cdot \cos \left(\frac{\pi \cdot i \cdot(2 \cdot j+1)}{2 \cdot X_{\max }}\right)\right),
$$

The data set $\left(S_{0}, X_{\max }, F F T N_{0} \div F F T N_{i}\right)$ taken from the etalon database.

Sample of saving etalon surface of the Chinese character "dragon" (Figure 4) by the first twenty harmonics of the Fourier series shown in Table 1.

Table 1: Example Description of Chinese Character "Dragon" in the Form of Normalized Values of the Fourier Series Harmonics in the Etalon Database

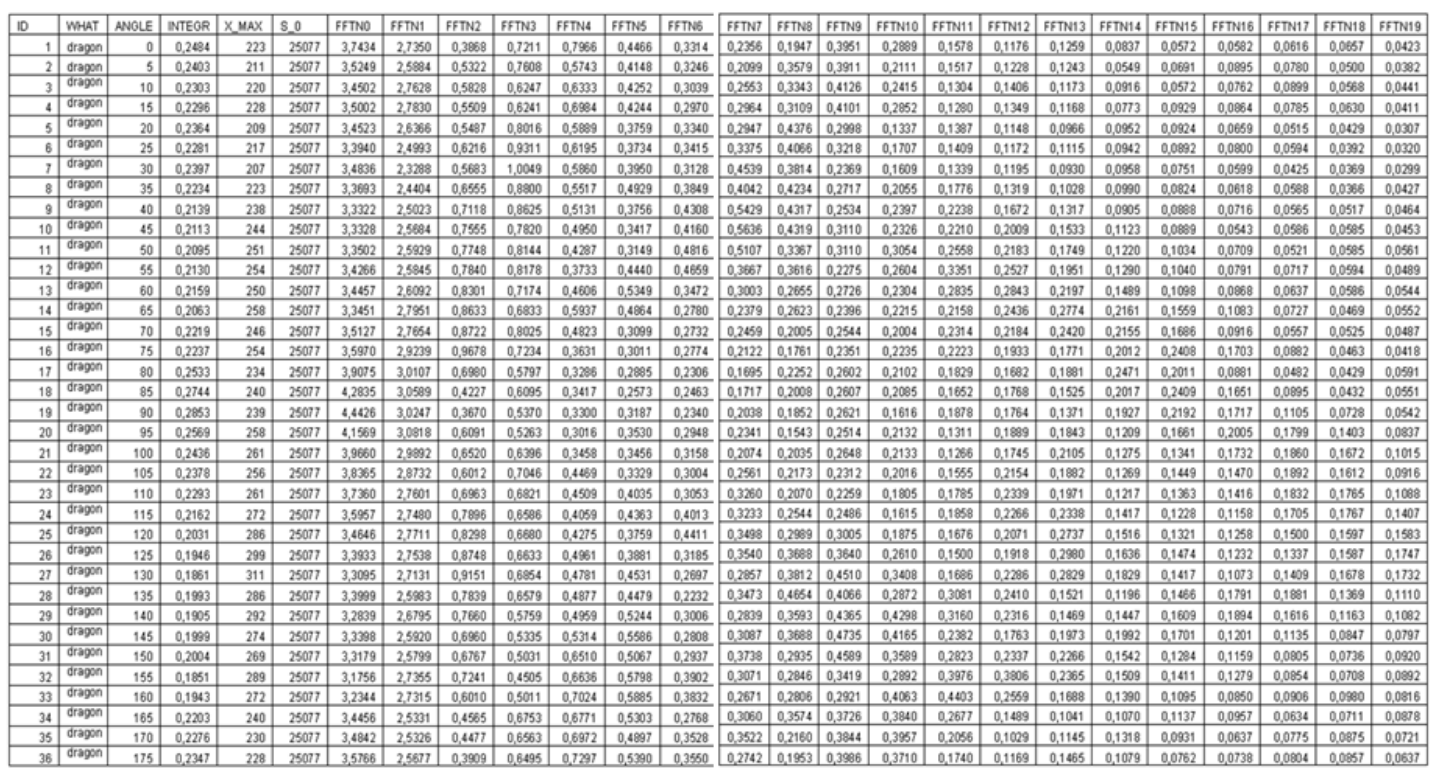

In the simulation program etalon surface of image described by normalized harmonic Fourier series (FFTNO-FFTN19) for 36 directions (ANGLE) with step 5 degrees. It covers a range of directions from 0 to $\pi$ which needed for the etalon saving. Also saved original values of the real image area (S_0) and maximal shift in this direction (X_MAX). The values of these two parameters correspond to the image for which carried out a training. The maximum shift is calculated in this case, in pixels, and the initial area equal to the number of pixels that belong to the training image. WHAT field contains the name of the object, and the field INTEGR is the value of the integral coefficient for the corresponding direction. It is used to accelerate the various image processing $[3,4]$.

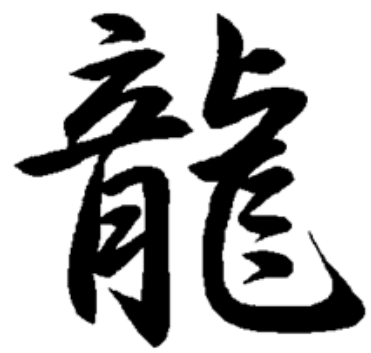

Figure 4: Image of Chinese character "dragon"

We can use for the reverse transformation not all Fourier series, but only a part of it, for a certain approximation to the shape of the input function. It is similar to the process of low-frequency filtration. The software simulation revealed that for the creation etalon FAl of convex shapes rather 
Stepan Bilan, Sergey Yuzhakov, Sergii Bilan; Saving of Etalons in Image Processing Systems Based on the Parallel Shift Technology. Advances in I mage and Video Processing, Volume 2 No 6, Dec (2014); pp: $36-41$

use the first five Fourier harmonics. For the creation etalon FAI of not convex shapes rather use the first ten Fourier harmonics. To create the etalon FAl of images which consist of several objects enough to use the first twenty Fourier harmonics. In all these cases the deviation of the restored FAI and real FAl does not exceed $10 \%$ of the real value of the function at each step shift. Also, when comparing the functions, you can use mean square deviation the real FAI and etalon FAI for a specific functions plot.

We can simulate the forgetting process with saving of etalon functions as Fourier series. Suppose that after the learning process the FAl described by the full set of the Fourier series harmonics. The number of elements of the series will coincide with the length of digitized function. The harmonic with the largest number can be removed from the etalon at regular intervals. Some information about the etalon to be lost, but will decrease the data which required to saving. Repeated recovery of the object becomes necessary when a function is based on the current number of harmonics will differ from the original by more than a specified value. Formula 3 turns to the next when the selected number of harmonics of the Fourier series $(\mathrm{NH})$ to restore the FAI.

$$
F A I(j)=S_{0} \cdot\left(\frac{F F T N_{0}}{\sqrt{X_{\max }}}+\frac{\sqrt{2}}{\sqrt{X_{\max }}} \sum_{i=1}^{N H-1} F F T N_{i} \cdot \cos \left(\frac{\pi \cdot i \cdot(2 \cdot j+1)}{2 \cdot X_{\max }}\right)\right),
$$

For example, for the image from the Figure 4 the FAl charts when using a certain number of the first Fourier series harmonic are as follows (Figure 5).

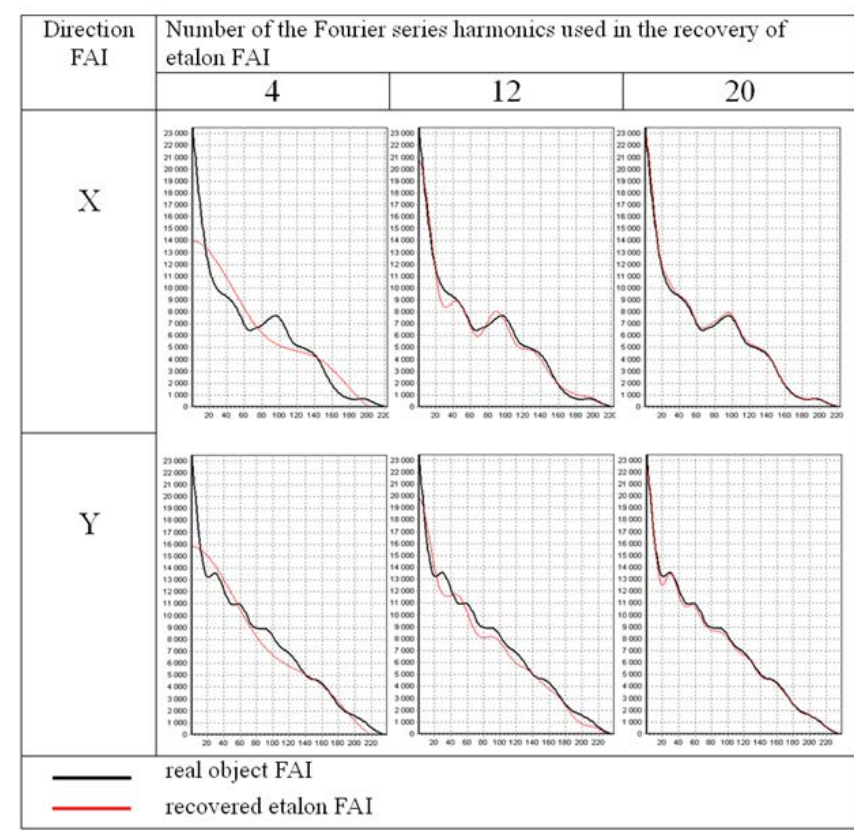

Figure 5: The charts of real object FAl and recovered etalon FAI

\section{Conclusion}

Saving of etalon information as functions can significantly reduce the amount of storage data. Image processing using the function of the area of intersection will speed the recognition process, the definition of spatial orientation and the movement parameters of the 2D-objects. However, the images recovery process with help of etalon surface is very complex.

The principles of manipulation with the 2D-objects can be used for the analysis of higher dimension objects. In this case, we must have the detectors intersection of objects with their copies. 
The objects of image processing research based parallel shift technology were the 2D binary image. Future plans to conduct research of processing grayscale images and scene analysis.

\section{REFERENCES}

[1]. Castillo O., Mellin P. Hybrid Intelligent Systems, Heidelberg (Germany): Springer, 2006.

[2]. Gonzalez R.C., Woods R. E., Eddins S. L. Digital Image Processing using MATLAB. Pearson Prentice-Hall, 2004.

[3]. Belan S., Yuzhakov S., A Homogenous Parameter Set for Image Recognition Based on Area, Computer and Information Science, 2013. 6(2): p. 93-102.

[4]. Belan S., Yuzhakov S., Machine Vision System Based on the Parallel Shift Technology and Multiple Image Analysis, Computer and Information Science, 2013. 6(4): p. 115-124. 